\title{
Ästhetik-Konzept zur Verbesserung der Hautqualität
}

Der Wunsch natürlich zu altern steht für viele Patienten im Vordergrund einer minimalinvasiven, ästhetischen Behandlung. Mit zunehmendem Alter nimmt der körpereigene Anteil an Hyaluron ab. Das Hyaluron in den Restylane Skinboostern wurde durch einen patentierten Herstellungsprozess stabilisiert und zeigt daher eine längere Haltbarkeit verglichen mit natürlichem Hyaluron in der Haut. Dies sorgt für eine langanhaltende, großflächige Hydratation bis in die tiefen Hautschichten. So wird die Elastizität der Haut gesteigert und feine Linien und Fältchen reduziert.

Das Ergebnis: natürlich wirkender, frischer Teint, eine sichtbar verbesserte Hautqualität und eine signifikante Verbesserung der Hautelastizität. Mit der
Anwendung können Hautunebenheiten, wie beispielsweise eingesunkene Aknenarben, ebenso sichtbar reduziert werden. Das spiegelt sich auch in der Kundenzufriedenheit wider:

- 3,5 Millionen Skinbooster-Behandlungen wurden bereits weltweit durchgeführt.

- Die Restylane Skinbooster basieren auf 12 Jahren klinischer Erfahrung.

- Nach der ersten Behandlung würden $91 \%$ der Patienten die Behandlung fortsetzen und $96 \%$ diese weiterempfehlen.

- Patienten sind nach Abschluss einer Behandlung mit dem Ergebnis zu $100 \%$ zufrieden.

- Langfristige Arzt-Patienten-Bindung: Das Aufteilen des Injektionsvolumens ist nicht nur angenehm für den Patienten, ein schrittweise klinisch fundiertes Behandlungsprotokoll kann auch die Patientenbeziehung stärken. Die Marktforschung zeigt: Zufriedenere Kunden sind loyalere Kunden.

Bei der Anwendung der Restylane Skinbooster kann der Behandler individuell nach dem vorliegenden Deckgewebe des Patienten entscheiden, welches Präparat für die Injektion geeignet ist. Für dünnere Haut mit wenig Gewebeabdeckung eignet sich die Verwendung von Restylane Skinboosters Vital Light. Für mehr Gewebeabdeckung hat der Behandler die Möglichkeit Restylane Skinboosters Vital anzuwenden.

Die Skinbooster sind für die Behandlungsbereiche im Gesicht, am Hals, an den Händen sowie am Oberarm und Dekolleté klinisch geprüft und stehen in zwei verschiedenen Applikationsformen zur Verfügung.

Nach Informationen von Galderma

\section{Verbesserung in der Therapie von Krampfaderleiden}

Krampfadern zählen zu den am meisten verbreiteten Krankheiten. 23 europäische phlebologische Gesellschaften sind sich einig und empfehlen die minimalinvasive Sklerotherapie zur Behandlung von kleinen Varizen in den europäischen Leitlinien als Goldstandard.

Auch das UK National Institute for Health and Care Excellence (NICE) veröffentlichte im Juli 2013 eine neue nationale Leitlinie, um eine evidenzbasierte Behandlung von Krampfadern zu fördern. In der NICE-Leitlinie zur Therapie von Krampfadern, die aus medizinischer Sicht einer Behandlung bedürfen, orientiert sich der Erfolg der Behandlung in erster Linie an der Verbesserung der Beschwerden, einer erhöhten Lebensqualität sowie am Verschwinden der Krampfadern. Therapieoptionen für Krampfadern der Stammvenen sind die thermischen Verfahren (endovenöse Laser- und Radiofre-

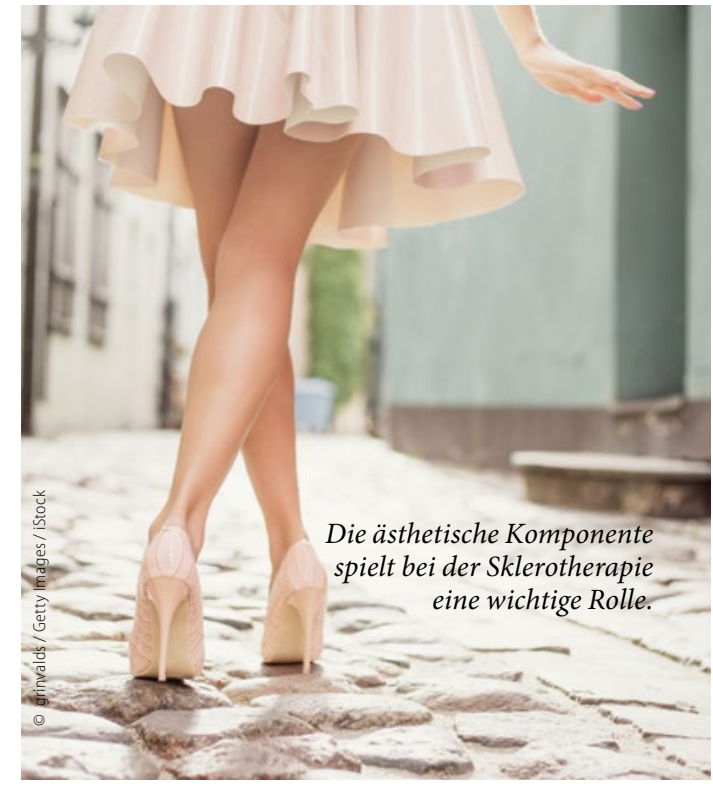

und findet keine relevanten klinischen Unterschiede. Insgesamt schnitten nach der Einschätzung der Autoren die thermischen Verfahren leicht positiver ab, wobei die Sklerotherapie die kostengünstigste aller Methoden ist. Dabei wird der Sklerotherapie ein höherer Stellenwert zugesprochen und laut NICE soll bei der Behandlung von Stammvarizen die SchaumSklerotherapie vor der operativen Methode empfohlen werden.

Eine aktuelle Studie belegt nun den Erfolg der NICE-Leitlinen in Großbritannien: Aufgrund dieser Empfehlungen konnte eine effektive Verbesserung in der Therapie von Krampfaderleiden erreicht und die Anwendung der kosteneffektivsten Therapie - der

quenztherapie), die Schaum-Sklerotherapie und die operativen Verfahren wie Stripping und Crossektomie. Die Leitlinie bewertet insgesamt alle Behandlungsmethoden als in etwa gleich gut wirksam
Schaum-Sklerotherapie - signifikant erhöht werden.
Nach Informationen von Chemische Fabrik

Kreussler \& Co 